\title{
1 The genomic landscape of contemporary western Remote Oceanians
}

2

Lara R. Arauna ${ }^{\mathrm{a}, 1}$, Jacob Bergstedt ${ }^{\mathrm{a}, \mathrm{b}, \mathrm{c}}$, Jeremy Choin ${ }^{\mathrm{a}, \mathrm{d}, \mathrm{e}}$, Javier Mendoza-Revilla ${ }^{\mathrm{a}}$, Christine Harmant ${ }^{\mathrm{a}}$, Maguelonne Roux ${ }^{\mathrm{a}, \mathrm{f}}$, Alex Mas-Sandoval ${ }^{\mathrm{g}}$, Laure Lémée ${ }^{\mathrm{h}}$, Heidi Colleran ${ }^{\mathrm{i}}$, Alexandre François ${ }^{\mathrm{j}}$, Frédérique Valentin ${ }^{\mathrm{k}}$, Olivier Cassar ${ }^{1}$, Antoine Gessain ${ }^{1}$, Lluis Quintana-

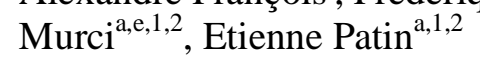

${ }^{a}$ Human Evolutionary Genetics Unit, Institut Pasteur, UMR 2000, CNRS, Paris 75015, France ${ }^{\mathrm{b}}$ Institute of Environmental Medicine, Karolinska Institutet, Stockholm 171 77, Sweden ${ }^{\mathrm{c}}$ Department of Medical Epidemiology and Biostatistics, Karolinska Institutet, Stockholm 171 77, Sweden

${ }^{\mathrm{d}}$ Université de Paris, Paris 75015, France

${ }^{\mathrm{e}}$ Chair Human Genomics and Evolution, Collège de France, Paris 75005, France

${ }^{\mathrm{f}}$ Bioinformatics and Biostatistics Hub, Institut Pasteur, Université de Paris, Paris 75015,

France

${ }^{\mathrm{g}}$ Department of Life Sciences, Imperial College London, Ascot SL5 7PY, United Kingdom

${ }^{\mathrm{h}}$ Biomics Platform, Institut Pasteur, Paris 75015, France

${ }^{\mathrm{i}}$ BirthRites Independent Max Planck Research Group, Department of Human Behavior, Ecology and Culture, Max Planck Institute for Evolutionary Anthropology, Leipzig 04103, Germany

${ }^{\mathrm{j}}$ Langues, Textes, Traitements Informatiques, Cognition (LaTTiCe), UMR 8094, CNRS, Paris 75015, France

${ }^{\mathrm{k}}$ MSH Mondes, UMR 7041, CNRS, Nanterre 92023, France

'Oncogenic Virus Epidemiology and Pathophysiology Unit, Institut Pasteur, UMR 3569,

CNRS, Paris 75015, France

${ }^{1}$ To whom correspondence may be addressed. Email: lrubioar@pasteur.fr (L.R.A.); quintana@pasteur.fr (L.Q.-M.); epatin@ pasteur.fr (E.P.)

${ }^{2}$ L.Q.-M. and E.P. contributed equally to this work.

Classification: Biological Sciences, Anthropology

Keywords: Pacific, populations, migrations, admixture, genetics, cultural practices

\section{This PDF file includes:}

Main Text

Figures 1 to 4 


\begin{abstract}
40 Abstract
41 The Vanuatu archipelago served as a gateway to Remote Oceania during one of the most 42 extensive human migrations to uninhabited lands 3,200 years ago. Ancient DNA studies 43 suggest an initial settlement by East Asian-related peoples that was quickly followed by the 44 arrival of Papuan-related populations, leading to a major population turnover. Yet, there is 45 uncertainty over the population processes and the sociocultural factors that have shaped the 46 genomic diversity of ni-Vanuatu, who present nowadays among the world's highest linguistic and cultural diversity. Here, we report genome-wide data for 1,433 contemporary ni-Vanuatu 48 from 29 different islands, including 287 couples. We find that ni-Vanuatu derive their East 49 Asian- and Papuan-related ancestry from the same source populations and descend from relatively synchronous admixture events that occurred $\sim 1,700-2,300$ years ago, indicating a peopling history common to all the archipelago. However, our analyses reveal that the Papuan population turnover was geographically uneven, and that the genetic contribution of Papuanrelated peoples was male-biased. Furthermore, we detect Polynesian ancestry arriving $\sim 600$ 1,000 years ago to South Vanuatu, and map its distribution to both Polynesian- and nonPolynesian-speaking islands. Lastly, we provide evidence for a tendency of spouses to carry similar genetic ancestry, when accounting for relatedness avoidance. The signal is not driven by strong genetic effects of specific loci or trait-associated variants, suggesting that it results instead from social assortative mating. Altogether, our findings provide insight into both the genetic history of ni-Vanuatu populations and how sociocultural processes have shaped the diversity of their genomes.

\section{Significance statement}

63 Vanuatu served as a gateway for the peopling of Remote Oceania but how demography and 64 culture have shaped the genomic diversity of its peoples remains partially understood. Here, 65 we report genomic data for 1,433 contemporary ni-Vanuatu covering 29 islands. We demonstrate that their genomic diversity derives from relatively synchronous, geographically uneven and sex-biased admixture events between the Austronesian-related first settlers and the Papuan-related subsequent incomers. We show that the more recent arrival of Polynesian ancestry did not always trigger shifts to Polynesian languages. Lastly, we find evidence for non-random mating, probably owing to social assortative mating. Our findings reveal the impact of multiple migrations, admixture and sociocultural practices on the genomic landscape of a key region of the South Pacific.
\end{abstract}




\section{$74 \quad$ Introduction}

Vanuatu, an archipelago located in western Remote Oceania, is a key region for understanding the peopling history of the Pacific. The cultural, anthropological and genetic diversity of Vanuatu reflects three distinct phases of population movements. The first, which started in present-day Taiwan 5,000 ya, was associated with the spread of Austronesian languages to Near and Remote Oceania (1-3). The so-called Austronesian expansion led to the emergence of the well-characterized Lapita cultural complex, which emerged in the Bismarck Archipelago and reached Vanuatu 3,200 ya $(4,5)$. Morphometric and ancient DNA (aDNA) studies indicate that the Lapita people in Vanuatu carried East Asian ancestry, supporting a connection with the Austronesian expansion $(6,7)$. The second migration occurred at the end of the Lapita period, 2,500 ya, and involved the arrival of Papuan-related peoples who shared ancestry with contemporary Bismarck Archipelago islanders. aDNA studies have shown that these migrations triggered a dramatic shift in genetic ancestry, from the East Asian-related ancestry associated with the Lapita culture to the Papuan-related ancestry that has remained predominant since then $(8,9)$. Finally, it has been postulated that "Polynesian outliers" from Vanuatu (i.e., Polynesian speakers living outside the Polynesia triangle) are the descendants of migrations from Polynesia into western Remote Oceania $(10,11)$, a model that has received recent support (12).

Although aDNA studies have revealed that ni-Vanuatu are descended from at least three ancestral populations $(8,9,12)$, whether the settlement process was uniform across the multiple islands of the archipelago remains an open question. aDNA data indicate that individuals dated to $\sim 2,500$ ya from South and Central Vanuatu carried largely different proportions of East Asian-related ancestry $(8,9,12)$, in line with either a unique population turnover that was geographically heterogeneous or separate admixture events between different populations across islands. Furthermore, Vanuatu is the country with the world's largest number of languages per capita (13), which supports the view that languages have rapidly diversified since the initial settlement and/or that the arrival of new groups to the archipelago further increased linguistic diversity. Nevertheless, these questions have been difficult to resolve because, to date, available ancient and modern DNA data from the region have remained sparse $(8,9)$.

Here, we generated genome-wide genotype data for 1,433 contemporary ni-Vanuatu and assessed their fine-scale genetic structure, in order to address central questions about the settlement of western Remote Oceania: do all contemporary ni-Vanuatu derive their ancestry from three populations only? Was the contribution of these three ancestral populations 
108 different across the archipelago? Was the late-Lapita shift to Papuan-related ancestry

109 heterogeneous across islands? In addition, the recent settlement of Vanuatu allows for the

110 study of how socio-cultural practices have shaped genetic diversity in humans over the past

$111 \sim 3,000$ years, in the form of sex-biased admixture, relatedness avoidance and non-random

112 mating (14-16). We thus used the comprehensive set of population genomics data presented

113 here, which includes 287 male-female couples, to answer important questions relating to the

114 genetic history of a population that descends from diverse ancestral populations: Was

115 admixture sex-biased? Was admixture accompanied by language shifts? Has socio-cultural

116 structure influenced mating? Can residence rules and urbanization affect human genetic

117 structure?

118 


\section{Results}

The Genetic Variation of Ni-Vanuatu is Spatially Structured. To shed light on the genetic make-up of ni-Vanuatu, we collected $>4,000$ blood samples from contemporary individuals between 2003 and 2005, and genotyped a selection of 1,433 of these sampled individuals at 2,358,955 single nucleotide polymorphisms (SNPs). After quality filters and merging with high-coverage whole genome sequencing data (17), 1,439 ni-Vanuatu samples were kept, including those from 522 males and 917 females living in 29 islands and 179 different villages (Fig. $1 A$ and Table S1).

Principal Component (PCA) and ADMIXTURE analyses indicate that ni-Vanuatu fall on a genetic gradient between East Asian-related and Papuan-related populations (Fig. $1 B$ and Fig. S1), supporting the view that their ancestry derives from these two population groups (6, $8,9,12)$. Furthermore, ni-Vanuatu show high genetic similarities with individuals from the Bismarck Archipelago, in line with the hypothesis that their Papuan-related ancestors originated from these islands $(6,8,9,12,17)$. However, the extensive geographic coverage of the dataset presented here enabled us to reveal substantial genetic substructure among niVanuatu islanders. PCA showed that genetic variation in ni-Vanuatu is explained by two contiguous but distinct groups that reflect geography (Fig. 1C and Fig. S2). Accordingly, when considering the number of ancestral populations that is most supported by ADMIXTURE $\left(K_{\mathrm{ADM}}=10\right)$, populations from different islands also show different ancestral components (Fig. S1). These results reveal that contemporary ni-Vanuatu show substantial genetic differentiation (Fig. S3), which could result either from different admixture histories during the settlement period and/or from the existence of barriers to gene flow that formed after the settlement of the archipelago.

To gain further insights into the genetic history of ni-Vanuatu, we next assessed the finescale genetic structure of the 1,439 sampled individuals, using ChromoPainter and fineSTRUCTURE (18-20). Haplotype-based clustering revealed a first separation between niVanuatu living north and south of the strait separating Epi and Tongoa islands $\left(K_{\mathrm{FS}}=2\right.$; Fig. S4). At $K_{\mathrm{FS}}=4$, populations separate into four clusters, here referred to as North, South, Central East and Central West Vanuatu clusters (Fig. 2A and Fig. S4 and Table S2), in general agreement with the classification of Oceanic languages spoken in the archipelago (21-23). Remarkably, individuals from the north and south of Pentecost island cluster with individuals from different neighboring islands, indicating that the sea does not necessarily act as a barrier to gene flow in the region, in line with linguistic and ethnographic data that reveal cultural networks between islands $(21,22,24,25)$. At $K_{\mathrm{FS}}=20$, the highest $K_{\mathrm{FS}}$ value for which 
statistical robustness remains maximal (Material and Methods) (19), we found that genetic clusters are often island-specific (Fig. 2A, Figs. S4-S7 and Table S1). These observations suggest that clusters inferred by fineSTRUCTURE are reliable, as they reflect expected geographic and linguistic barriers to gene flow.

\section{The Late-Lapita Shift to Papuan-related Ancestry was Geographically Uneven. We} detected important differences among fineSTRUCTURE clusters in the proportions of East Asian-related ancestry, that is, ancestry related to contemporary populations from either Taiwan and the Philippines or Polynesia (range of SOURCEFIND estimates: 0.15 to 0.55 ; Fig. 2C, Figs. S8-S11 and Table S3). East Asian-related ancestry was found to be lowest for the Central Vanuatu clusters (e.g., Malekula, Ambrym, Epi; median $=0.230, \mathrm{SD}=0.056$ ) and highest in the South Vanuatu cluster (i.e., Efate, Tongoa, Ifira and Emae islands; median = 0.323, SD = 0.077), where "Polynesian outlier" communities live today (10). Nevertheless, East Asian-related ancestry is also high in islands where Polynesian ancestry is low, such as Ambae (median $=0.405, \mathrm{SD}=0.042 ;$ Fig. $2 D$ ), suggesting that differences in East Asianrelated ancestry are not due solely to differences in Polynesian ancestry (Figs. S10 and S11 and Table S3).

To assess whether differences in East Asian-related ancestry originate from a geographically uneven ancestry turnover or separate admixture events between distinct populations, we dated admixture in each genetic cluster separately. Besides more recent events relating to Polynesian migrations (see next section), all estimates overlap the same time period that ranges from 1,700 to 2,300 ya (Fig. 2B, Fig. S12 and Table S4), suggesting that all ni-Vanuatu share the same admixture history. To test this hypothesis more formally, we evaluated if the Papuan-related ancestry carried by present-day ni-Vanuatu derives from a single source. PCA and $f_{4}$-statistics of the form $f_{4}(X$, New Guinean highlanders; Solomon or Bismarck Archipelago islanders, East Asians) indicate that all ni-Vanuatu show similar genetic relatedness with populations from New Guinea, the Bismarck Archipelago or the Solomon islands (Figs. S13 and S14), in agreement with aDNA data (12). Furthermore, the haplotype-based SOURCEFIND method detected that the same cluster of Bismarck Archipelago islanders is the source that contributed the most to all ni-Vanuatu (Fig. S15 and Table S5). Of note, SOURCEFIND also detected a small contribution from New Guinean highlanders and Santa Cruz islanders in all ni-Vanuatu clusters, which negatively correlates with Polynesian ancestry $\left(r=-0.378, P\right.$-value $\left.<2.22 \times 10^{-16}\right)$, probably because Polynesian source populations capture some Papuan-related ancestry. Collectively, these findings 
indicate that ni-Vanuatu are descended from relatively synchronous admixture events between the same sources of Papuan- and East Asian-related ancestry; yet, their proportions differ markedly across islands, indicating that the dramatic Papuan-related ancestry shift that started $\sim 2,500$ ya was geographically uneven.

\section{Polynesian Migrations Did Not Necessarily Trigger Language Shifts. Admixture}

proportions and date estimates indicate that ni-Vanuatu ancestry partly derives from a third and more recent migration originating from Polynesia (Fig. $2 B-D$ and Tables S3 and S4). We dated admixture events between 600 and 1,000 ya for genetic clusters predominant in Efate, Ifira, Emae and Futuna islands (Fig. 2B, Fig. S12 and Table S4), where Polynesian languages are spoken today (26). These clusters show higher Polynesian ancestry and, therefore, higher East Asian-related ancestry proportions, relative to North and Central Vanuatu clusters (SOURCEFIND estimates; Fig. 2C-D, Figs. S8-S11 and Table S3). Ni-Vanuatu Polynesian speakers show a higher ratio of Polynesian-to-East Asian ancestry, when compared to nonPolynesian speakers (ratio $=0.647$ vs. 0.479 ; Wilcoxon test $P$-value $<2.22 \times 10^{-16}$; Fig. $2 D$ and Fig. S10). Furthermore, $f_{4}$-statistics of the form $f_{4}(X$, East Asians; Tongans, New Guinean highlanders) suggest that ni-Vanuatu from Futuna, Ifira, Efate, Emae and Tongariki share more alleles with Polynesians than other ni-Vanuatu do (Fig. S16).

Interestingly, our analyses also revealed that Polynesian ancestry is not restricted to Vanuatu islands where Polynesian languages are spoken. Non-Polynesian-speaking populations assigned to South Vanuatu (e.g., Tongoa, Tongariki and Tanna) and North Vanuatu (i.e., Banks and Torres islands) clusters also show a higher Polynesian-to-East Asian ancestry ratio than Central East and West ni-Vanuatu (ratio $=0.557$ and 0.510, vs. 0.461; Wilcoxon test $P$-value $<2.22 \times 10^{-16}$; Fig. $2 D$, Figs. S10 and S11 and Table S3). Furthermore, estimated admixture dates are similar among South Vanuatu clusters where both Polynesian and non-Polynesian languages are spoken (Fig. 2B, Fig. S12 and Table S4). These results support the view that Polynesian migrations, as well as subsequent contacts between Vanuatu islands $(26,27)$ or with "Polynesian outliers" from the Solomon islands (28), also introduced Polynesian ancestry among non-Polynesian-speaking groups.

Conversely, we found no evidence of admixture with Polynesians in individuals assigned to Central Vanuatu clusters, including Epi islanders, despite the geographic proximity between Epi and Tongoa (Fig. 2D, Figs. S10 and S11 and Table S3). Of note, ADMIXTURE, PCA and fineSTRUCTURE analyses separate ni-Vanuatu into northern and southern populations, the frontier between the two being located between Epi and Tongoa (Figs. $1 C$ 
and $2 A$ and Fig. S1). The strait that separates the two islands today is the location of the Kuwae caldera $(29,30)$, which may have been a barrier to gene flow. Together, these results reveal that, since 1,000 ya onward, Polynesians migrated to Vanuatu where they admixed with local populations, and that such interactions did not necessarily result in a shift to Polynesian languages.

A Limited Genetic Impact of European Colonization. Despite the relatively long history of European colonization of Vanuatu, which started in $1606 \mathrm{CE}(31,32)$, our genetic data indicate that admixture between ni-Vanuatu and Europeans has been rare or has left few descendants in Vanuatu. In total, only 28 individuals out of 1,439 (1.95\%) show a genetic contribution from Europeans higher than 1\%, according to SOURCEFIND analyses (range: $0.03 \%$ to $35.3 \%$; Fig. S8 and Table S3). The two fineSTRUCTURE clusters with the highest European ancestry (median $=0.093$ and $0.107, v s .0 .002$ for other clusters) show an admixture event in the last 120 years (mean $=78$ ya, 95\%CI: [74-81] and mean $=111$ ya, 95\%CI: [104118]) (Fig. 2B and Table S4). Similarly, the three other clusters that include individuals carrying more than $1 \%$ of European ancestry show a pulse of admixture occurring in the last 200 years. These results are consistent with historical records, which indicate that contacts between Europeans and ni-Vanuatu were initially rare but became more common when Christian missionaries and colonists settled in the archipelago during the $19^{\text {th }}$ century, when the first intermarriages were reported $(31,32)$.

Genetic Admixture in Vanuatu was Sex-Biased. Genetic studies have suggested that Papuan-related migrations from the Bismarck Archipelago into Remote Oceania were malebiased, because contemporary Polynesians and a few ancient individuals from present-day Vanuatu show lower Papuan-related ancestry on the X chromosome, relative to autosomes (6, 12). To confirm that admixture between the ancestors of present-day ni-Vanuatu was sexbiased, we estimated Papuan- and East Asian-related ancestry in ni-Vanuatu on each chromosome separately, using local ancestry inference (33). We found that Papuan-related ancestry is indeed significantly lower on the $\mathrm{X}$ chromosome, relative to autosomes $\left(\alpha_{\mathrm{X}}=\right.$ $75.2 \%$ vs. $\alpha_{\text {auto }}=79.8 \%$, Wilcoxon test $P$-value $<1.36 \times 10^{-5}$; Fig. $3 A$ ), confirming aDNA results (12). These values were similar between Polynesian and non-Polynesian speakers (Fig. S17A, Wilcoxon test $P$-value $=0.13$, which indicates that it is not explained by recent migrations from Polynesia. We replicated the results using high-coverage genome sequences from a subset of 179 ni-Vanuatu (17), indicating that our results are not biased due to SNP 
ascertainment (Fig. S17B). Assuming that admixture proportions have reached equilibrium values (34), we estimated that the genetic contribution of Papuan-related males to ni-Vanuatu was $27 \%$ higher than that of Papuan-related females $\left(\alpha_{\mathrm{m}}=93.5 \%\right.$ vs. $\left.\alpha_{\mathrm{f}}=66.1 \%\right)$.

Accordingly, Y chromosomes of ni-Vanuatu are dominated by haplogroups found at high frequency in Near Oceanians (e.g., M1a3b2, S1), whereas mitochondrial DNAs (mtDNA) show a high proportion of haplogroups typically found in East Asia (e.g., B4a1a1, E1a2a4) (Fig. $3 B$ and Table S1). Collectively, these results indicate that ni-Vanuatu ancestry predominantly results from admixture between Papuan-related males and East Asian-related females.

Recent Migrations are Influenced by Residence Rules and Urbanization. The genetic structure of contemporary ni-Vanuatu is also expected to reflect socio-cultural practices (e.g., social networks, exchange and marriage rules) that have culturally evolved since the settlement of the archipelago. We leveraged the high-resolution genetic data to infer recent migrations between Vanuatu islands - indicated by individuals who inhabit an island but belong to a genetic cluster that is prevalent in another island - and determine if these migrations have involved mainly females or males, in line with virilocal or uxorilocal postmarital residence rules, respectively. We found that $5.70 \%$ of the sampled individuals (54 individuals) migrated at a large geographical scale $\left(K_{\mathrm{FS}}=4\right)$, while $11.81 \%$ (112 individuals) migrated at a local scale $\left(K_{\mathrm{FS}}=20\right.$; Table S6), suggesting more genetic connections between closer islands. We estimated that local mobility among females is higher than among males $\left(K_{\mathrm{FS}}=20\right.$; odds ratio $=2.20$, Fisher's exact test $P$-value $=9.16 \times 10^{-4} ;$ Fig. $3 C$ ), as expected under virilocal residence and/or female exogamy (35). The same trend was observed at a larger geographical scale, when considering migrations between four broader regions $\left(K_{\mathrm{FS}}=\right.$ 4 ; odds ratio $=1.86, P$-value $=0.075)$ and when restricting the analyses to the reported birth place of male-female couples included in the dataset $($ Fig. S18) $($ odds ratio $=1.96, P$-value $=$ $\left.6.89 \times 10^{-3}\right)$. Notably, comparisons of the places of birth and residence of sampled individuals did not support female-biased migrations (odds ratio $=0.807, P$-value $=0.18$; Fig. S19 and Table S6), possibly reflecting a bias in the self-reported birth places. This might occur if women are inclined to report their husband's or children's place rather than their father or mother's place (36).

We also explored the direction of the inferred migrations, and found that both female and male migrations mainly occurred from the northernmost and southernmost islands towards the center of the archipelago (Fig. 3C), where Port Vila - the largest city - has developed (37). 
Migrations are also common among Central Vanuatu islands, consistent with a long-term network of cultural and material exchanges in the region (28). Thus, our genomic data reflect the migration patterns that characterize the recent history of ni-Vanuatu, including residence rules and urbanization.

Evidence for Relatedness Avoidance. Human kinship systems vary tremendously, regulating marriage, exchange, endogamy or exogamy according to indigenous relational concepts (38). Given the small census size of ni-Vanuatu populations, it is interesting to consider whether and how sociocultural features like marriage and exchange rules influence local levels of genetic relatedness. We found that genetic relatedness was higher within islands than between islands (Wilcoxon test $P$-value $<2.22 \times 10^{-16}$; Figs. S20-S22), consistent with an isolation by distance model (Mantel test $P$-value $=0.001 ; 1,000$ permutations). Accordingly, out of the 287 male-female couples included in the dataset, $78.4 \%$ were born on the same island (Fig. S23). Genetic relatedness is also higher among inhabitants of the same village, relative to individuals living on the same island (Wilcoxon test $P$-value $<2.22 \times 10^{-16}$ ) (Fig. S20), suggesting that the local community is often the source of marriage partners. However, despite the generally high levels of genetic relatedness observed between niVanuatu, we did not observe an excess of genetic relatedness among couples (Figs. S23 and S24). Specifically, spouses tend to show slightly lower kinship coefficients than random pairs of individuals from the same village, after excluding first-degree related individuals (logistic regression model $P$-value $=0.057$; resampling $P$-value $=0.079)($ Figs. S24 and S25). These results indicate that the practice of exogamy has affected the genetic diversity of ni-Vanuatu, and provide a vivid example that human populations can show high levels of genetic relatedness in the absence of endogamous marriage practices.

Ni-Vanuatu Spouses Tend to Share Similar Genetic Ancestry. Research on admixed populations from other parts of the world has shown that, in addition to low genetic relatedness, partners tend to show similar genetic ancestry (15), because mating often occurs within socio-cultural groups, which can correlate, in turn, with genetic ancestry. To verify whether this phenomenon is observed in Vanuatu, we implemented a logistic regression model that jointly estimates the effects of geography, genetic relatedness and genetic ancestry on the probability to be partners (Material and Methods). These analyses confirmed that spouses tend to originate from the same island, while showing lower kinship coefficients than non-spouses (Fig. 4A). Importantly, we found that spouses show lower differences in genetic 
ancestry than non-spouses, when considering Papuan-related $(\beta=-4.233, P$-value $=0.018)$,

East Asian-related $(\beta=-4.221 P$-value $=0.018)$ or Polynesian $(\beta=-5.197, P$-value $=0.024)$ ancestry. These results suggest that ni-Vanuatu tend to mate with a partner who carries similar genetic ancestry.

Two hypotheses have been proposed to explain these observations. First, social assortative mating may underlie the observed signal, as genetic ancestry can correlate with socio-cultural structure. Second, spouses may choose their partner because they share biological traits, such as physical appearance $(39,40)$. To test these hypotheses, we searched for genomic loci that could play a role in partner choice, by including, in the logistic regression model, a term that measures dissimilarity between individuals at each SNP (Material and Methods). No SNP showed statistical evidence for a significantly lower or higher genotype similarity between spouses, when accounting for multiple testing (Fig. S26). To test for assortative mating according to polygenic traits, we then evaluated if genotype similarity between spouses is significantly higher or lower at SNPs associated with candidate traits relating to physical appearance, when compared to non-associated SNPs. When we did not account for genetic structure and ancestry-associated assortative mating (i.e., effect sizes are estimated from a model where these confounders are not included, see Material and Methods), we found evidence for assortative mating according to body mass index (BMI, Fig. 4B). However, when accounting for such possible confounders, we found no statistical evidence that genotypes at trait-associated variants are more similar or dissimilar between spouses than expected. Collectively, our results do not support a marked tendency for partner choice according to genetic or phenotypic features, and suggest instead the occurrence of assortative mating driven by social structure as the cause for ancestry-based assortments among niVanuatu. 


\section{Discussion}

349

350

351

352

353

354

355

By leveraging an extensive genomic dataset of 1,433 contemporary individuals, we show here that all ni-Vanuatu initially descend from admixture between the same ancestral populations: an East Asian-related population, which shares genetic affinities with groups living today in Taiwan and the Philippines, and a Papuan-related population, which shares genetic affinities with groups living today in the Bismarck Archipelago. We also find that admixture was relatively synchronous across islands, in agreement with a peopling history common to all the archipelago. Thus, our results suggest that the exceptionally high cultural diversity of niVanuatu results from a rapid cultural diversification that developed in situ, as suggested by linguistic, archaeological and archaeogenetic studies $(12,13,28)$. Nevertheless, our results cannot exclude a scenario where the islands were settled by multiple, closely related groups carrying East Asian-related or Papuan-related ancestry. Furthermore, we caution that admixture date estimates from modern DNA data are uncertain and can be biased downward when admixture was gradual (41), which was likely the case in Vanuatu (9). Additional aDNA time transects from multiple islands will be required to provide a definitive picture of the genetic history of ni-Vanuatu.

Our analyses reveal substantial differences in East Asian-related ancestry proportions between islands. We show that these differences do not result solely from Polynesian migrations, as our haplotype-based analyses could differentiate ancestry attributed to the Austronesian expansion from that introduced by Polynesians. A compelling example is Ambae, where East Asian-related ancestry is 1.8-times higher than in surrounding islands but where Polynesian ancestry is low. These findings indicate that the major population turnover following the arrival of Papuan-related peoples was geographically uneven, possibly because, at the time of admixture, the two ancestral populations of ni-Vanuatu were of different sizes across islands, some of which being preferentially settled by Austronesian-related groups and others by Papuan-related groups. Lastly, we find evidence that admixture was sex-biased; either Papuan-related migrants were predominantly males or both males and females migrated, but admixture was more common between Papuan-related males and East Asianrelated females.

A recent archaeogenetic study has reported that ancient ni-Vanuatu from Efate and Eretok show genetic similarities with Polynesians (12), supporting the occurrence of migrations from Polynesia, which were previously postulated by linguistic studies $(10,11)$. We confirm that "Polynesian outlier" communities in Vanuatu are descended from admixture events between Polynesians and local populations. We dated these admixture events to 600-1,000 ya, in line 
with archaeological records (42). Furthermore, we extend previous findings by mapping the genetic impact of Polynesian migrations to some Vanuatu islands where Polynesian languages are not spoken today (e.g., Makura, Tongoa, Tongariki and Tanna) (26). These results indicate that genetic interactions between ni-Vanuatu and Polynesian incomers did not systematically trigger shifts to Polynesian languages. Intriguingly, Polynesian ancestry is not detected north of the Kuwae caldera, a large submarine volcano that separates Tongoa and Epi islands. Geological data have shown that the Kuwae volcano erupted in ca. 1452, producing among the largest volumes of magma and aerosol ever recorded $(29,30)$. We suggest that this caldera, or other active volcanoes in the region, may have acted as a barrier to Polynesian gene flow. Nowadays, ni-Vanuatu living north and south of Kuwae form two genetic groups with distinct socio-cultural practices (e.g., grade-taking and chiefly title political systems) (43), indicating that the area has remained a genetic and cultural frontier. Besides these two groups, we observed subtle genetic differences among ni-Vanuatu, which recapitulate geography and known language subgroups. Yet, migration between islands is common, probably owing to long-term networks of exchange. An illustrative example is Pentecost island, where northern and southern groups show fewer genetic similarities than either do with groups from nearby islands.

By building upon the well-defined genetic history of Vanuatu, we also explored how genetic diversity has been shaped by cultural practices. While levels of genetic relatedness are high among ni-Vanuatu, we do not find evidence for generalized endogamy, which challenges the frequent association geneticists make between the two processes $(16,44-46)$. Nonetheless, even if ni-Vanuatu spouses are generally less related than non-spouses, we show that their genetic ancestries are more similar than expected, indicating that mating in Vanuatu is not random. Importantly, ancestry similarity between partners is not stronger at trait-associated SNPs, suggesting that ancestry-associated assortments are due to social structure, which may, in turn, be correlated with levels of East Asian-related and/or Polynesian ancestry. Other studies have suggested non-random mating according to ancestry in regions of the world where socio-cultural structure is highly correlated with ancestry $(15,47)$. Our findings extend the occurrence of such socio-cultural assortments to Oceanians, raising questions of how common this phenomenon is in human societies and whether non-random mating should systematically be accounted for in human genetic studies. Collectively, our study emphasizes the need to include diverse populations in genetic studies, not only to address key anthropological and evolutionary questions that are important for specific geographic regions, but also to identify factors shaping the genetic diversity of human populations as a whole. 


\section{Material and Methods}

\section{Sample collection and approvals}

418 The sampling survey was conducted in the Republic of Vanuatu between April 2003 and August 2005. The purpose of the study was the estimation of the seroprevalence of HTLV-1 viral infection and the assessment of human genetic diversity in ni-Vanuatu. The recruitment of participants was carried out after the agreement of the Ministry of Health of Vanuatu, the head of each Directorate from the sampled province, and the chief of the sampled village. The data collectors, Olivier Cassar, Helene Walter, Woreka Mera and Antoine Gessain, were accompanied by the village chief and/or the head of the local dispensary. The nature and the scope of the study were explained in detail by Olivier Cassar in English, and by Helene Walter in Bislama (i.e., an English-based Creole that is the main lingua franca of Vanuatu), during information meetings organized in each village. Participants could ask any questions after the information meeting. After several hours of reflection, each volunteer participant of at least 18 years of age was asked to sign a written informed consent form, including consent for research on human genetic diversity. Sex, age and birth place, as well as the date and place of blood collection, were collected through a structured questionnaire. Couples were identified through interviews in English or Bislama, and were preferentially sampled. Blood samples were collected either at the local dispensary, a gymnasium or a hut provided by the village chiefs.

The study received approval from the Institutional Review Board of Institut Pasteur ( $\left.{ }^{\circ} 2016-02 / \mathrm{IRB} / 5\right)$ and the Ministry of Health of the Republic of Vanuatu. It was conducted in full respect of the legal and ethical requirements and guidelines for good clinical practice, in accordance with national and international rules. Namely, research was conducted in accordance with: (i) ethical principles set forth in the Declaration of Helsinki (Version: Fortaleza October 2013), (ii) European directives 2001/20/CE and 2005/28/CE, (iii) principles promulgated in the UNESCO International Declaration on Human Genetic Data, (iv) principles promulgated in the Universal Declaration on the Human Genome and Human Rights, (v) the principle of respect for human dignity and the principles of non-exploitation, non-discrimination and non-instrumentalisation, (vi) the principle of individual autonomy, (vii) the principle of justice, namely with regard to the improvement and protection of health and (viii) the principle of proportionality. The rights and welfare of the subjects have been respected, and the hazards did not outweigh the benefits of the study.

\section{SNP genotyping and quality filters}


450 Five millilitres of blood were obtained by venepuncture from each volunteer participant and

451 transferred to the Institut Pasteur of New Caledonia, where plasma and buffy coats were

452 isolated, frozen, and stored at $-80^{\circ} \mathrm{C}$. Samples were then transferred to the Institut Pasteur in

453 Paris (France). Out of the 4,428 collected samples, 1,433 samples were selected for genetic

454 analyses. These samples were selected in order to (i) cover the largest number of islands and

455 villages, (ii) cover locations where "Polynesian outliers" live nowadays and (iii) include as

456 many couples as possible. After sample selection, a total of 179 different villages were

457 covered, located on 29 islands (Table S1). DNA was purified from frozen buffy coats at the

458 Institut Pasteur of Paris (France), using QIAamp DNA Blood Mini Kit protocol, and eluted in

459 AE buffer. DNA concentration was quantified with the Invitrogen Qubit 3 Fluorometer using

460 the Qubit dsDNA broad-range assay. Prior to SNP array genotyping, DNA integrity was

461 checked on agarose gels.

462 The 1,433 selected samples were genotyped on the Illumina Infinium Omni 2.5-8 array

463 (San Diego, California). Genotype calling was performed using the Illumina GenomeStudio

464 software. We excluded 2,491 SNPs with missing annotations, 9,661 duplicated SNPs, 1,772

465 SNPs with a GenTrain score < 0.4, SNPs with a missingness $>0.05$ and SNPs that deviate

466 from Hardy-Weinberg equilibrium (i.e., $P$-value $<0.01$ in more than one Vanuatu island).

467 Only autosomal SNPs were kept for the analyses, unless otherwise stated. After filters, a total

468 of 2,269,868 SNPs were kept. When the analyses required minor allele frequency (MAF)

469 filters, a MAF > 0.01 threshold was applied. When a linkage disequilibrium pruning was

470 required, we pruned the data using a window size of $50 \mathrm{~Kb}$, a step size of 5 SNPs and a $r^{2}$

471 threshold of 0.5. After all these filtering steps were applied, the remaining number of SNPs

472 was 294,806. All filters were applied using PLINK v.1.9b (48).

\section{Sample quality filters}

The highest genotype missingness per sample was 0.018 . We removed 21 samples with outlier values for heterozygosity (mean $\pm 3 \mathrm{SD}$ ), suggestive of DNA contamination, leaving 1,412 samples. Cryptic relatedness between samples was detected using KING v.2.1 (49). We excluded 456 samples with a kinship coefficient $>0.08$ with another sample, whenever analyses required unrelated samples. For 12 samples, the reported sex did not match the genetic sex inferred by the Y- and X-chromosome call rates. These include one couple, for which the reported sex is the opposite to the genetic sex. We thus exchanged the sex of the two samples, as it is most likely an error on the sample annotation. We did not include the 10 
remaining samples, when performing analyses relating to mating practices and sex-specific migrations. After quality filters, a total of 287 couples was present in the dataset.

\section{Demographic information}

Demographic data include the island of birth and the village of residence. Birth place was missing for 73 samples and the residence for 49 samples. From this information, we retrieved, for each sample, the geographical coordinates of the birth place (around the center of the island) and of the place of residence (around the village of residence), using MapAction reference maps (www.mapaction.org). We considered as the locations of "Polynesian outliers" all the villages within Futuna, the villages of Mele and Imere in Efate, Ifira island and the villages of Makatea, Tongamea and Vaitini in Emae (26). Among the 287 couples, 207 reported the same island of birth, while 57 reported a different island. This information was missing for 23 couples.

\section{Merging with reference datasets}

We merged the new SNP array data with whole genome sequences of different populations across the Pacific (17) and worldwide populations from the SGDP project (50). We also merged the dataset with SNP array data from Oceanian populations (41), to perform some population genetics analyses. Datasets were merged using PLINK v.1.9b (48). Transversions were excluded, to avoid allele strand inconsistences.

\section{Haplotype phasing}

We phased the dataset using SHAPEIT v.2 $(51,52)$ using 500 conditioning states, 10 burn-in steps, 50 Markov chain Monte Carlo (MCMC) main steps, a window length of $1 \mathrm{cM}$ and an effective population size of 15,000. We used 1000 Genomes data (53) as a reference panel and therefore removed all array SNPs that did not align with this data, prior to haplotype phasing.

\section{Genetic structure: PCA and ADMIXTURE}

We performed Principal Component Analyses (PCA) with the 'SmartPCA' algorithm implemented in EIGENSOFT v. 6.1.4 (54). aDNA samples were projected on PCs by using the "lsqproject" and "shrinkmode" options. We inferred population structure with ADMIXTURE v. 1.3.0 (55), using 10 different seeds and assuming that $K_{\mathrm{ADM}}$ varies from 2 to 17, and visualized ADMIXTURE results using pong v1.4.7 (56). We estimated pair-wise $F_{\mathrm{ST}}$ 
517 values using the StAMPP R package (57), and assessed their significance with 100 bootstraps.

518 We computed $f_{4}$-statistics with ADMIXTOOLS v.6.0 and estimated standard errors by block

519 jackknife.

520

\section{Genetic structure: ChromoPainter and fineSTRUCTURE}

We ran ChromoPainter (18) to infer haplotype sharing among individuals. This algorithm is based on a Hidden Markov Model in which each sample is treated as a "recipient", i.e., a mosaic of haplotypes from a set of "donor" samples. We performed different analyses with ChromoPainter. In Analysis 1, we inferred the genetic structure of ni-Vanuatu running ChromoPainter only for the unrelated ni-Vanuatu samples. We set each sample both as a recipient and as a donor for all the samples (-a mode). We first estimated the global mutation probability and the switch rate with 10 expectation-maximization (EM) iterations, by using the '-in -iM' options and by averaging estimates obtained for chromosomes 1, 7, 13 and 19. The estimated values were $1.542 \times 10^{-4}$ and 81.415 , respectively. Then, we ran ChromoPainter in the same mode for all chromosomes but fixing the parameters to estimated values. In Analysis 2, we repeated the same process independently for the reference populations. In this case, we ran ChromoPainter only for the samples outside Vanuatu $(17,50)$ and repeated the two-step process described above. In this case, the global mutation probability was estimated to $4.53 \times 10^{-4}$ and the switch rate was 255.42 .

We then produced a coancestry matrix by summing the coancestry matrices for all chromosomes and estimated the $C$ parameter with ChromoCombine. The $C$ factor was estimated at 0.452 for the Vanuatu dataset (Analysis 1), and 0.498 for the reference population dataset (Analysis 2). We applied the model-based Bayesian clustering method fineSTRUCTURE v.2.0.7 (18) on ChromoPainter coancestry matrices. We ran 1 million burn-in iterations (-y option) and 1 million MCMC iterations (-x option) and sampled values every 10,000 iterations. We then inferred the fineSTRUCTURE tree (-m T option). We repeated this process 5 times, with different random seeds.

Following a previous study (19), we estimated for each individual the robustness of the clustering assignation, by comparing the final state (i.e., the state with the highest posterior probability) with the 100 MCMC samples. For each individual $i$, we estimated $x_{i}^{(m)}$, the number of individuals clustering with $i$ for each of the $m=1, \ldots, 100$ MCMC samples. We also estimated $y_{i k}^{(m)}$, the number of individuals that cluster with $i$ in the final state and in each of the MCMC samples, for each inferred cluster $k$. Finally, we computed 
$P_{i}=\sum_{m}\left[y_{i k}^{(m)} / x_{i}^{(m)}\right]$. Therefore, we ended up with a matrix of this sum $P_{i}$, for all individuals and for each genetic cluster, that shows the robustness in the assignation of each individual to its corresponding cluster in the final state. We repeated this process for $k=2, \ldots, K, K$ being the maximum number of clusters identified by fineSTRUCTURE. We also repeated the process for the 5 random seeds and compared the performance of each seed. Fig. S6 shows the number of ni-Vanuatu individuals with $P_{i}<0.9$ for each seed $s$ and for each $k=2, \ldots K$. These estimations allowed to test the robustness in the cluster assignation of each individual, to compare the performance across seeds and to determine the robustness at each $k$ value.

To compare robustness among seeds, we followed a similar approach. We estimated $x_{i}^{(s)}$, where $s=1, \ldots, 5$, which corresponds to the number of individuals clustering with individual $i$ for each seed. We then estimated $y_{i k}^{(s)}$ and $\sum_{s}\left[y_{i k}^{(s)} / x_{i}^{(s)}\right]$, which shows the concordance in the assignation of each individual to a cluster across the different seeds. To summarize this information, we analyzed all the individual sums, for each seed and each cluster $k$ (Fig. S7A and $C$ ), as well as the number of individuals who were not assigned to the same cluster, between one seed and all the other seeds (Fig. S7B and $D$ ).

Based on these analyses, we chose the seed that showed the highest consensus among seeds (Fig. S7) and in which the largest number of samples are robustly assigned (Fig. S6) (seed number 289 for Analysis 1 and 161 for Analysis 2). Then, we defined the maximum $k$ value based on the number of individuals who are robustly assigned to a cluster for each $k$ value and we focused our analyses on this value. Finally, once we chose a seed and a $k$ value, we removed samples that showed some uncertainty in the cluster assignation $\left(P_{i} \leq 0.9\right)$. Following this process, we observed that the robustness decreases from $K_{\mathrm{FS}}=20$ and we removed 3 samples from Analysis 1 (Vanuatu). For the reference dataset (Analysis 2), all samples showed a $P_{i}>0.9$ across all $k$ values, therefore we used genetic clusters from the $k$ value that best suited the hypotheses to test $\left(K_{\mathrm{FS}}=25\right)$ (Table $\left.\mathrm{S} 5\right)$.

For the subsequent analyses, we redefined populations based on fineSTRUCTURE results and therefore on the genetic data itself. This approach is rather trivial when applied to continental populations, like the reference populations presented here, because fineSTRUCTURE clusters coincide broadly with the geographical or cultural categories assigned to the populations. Yet, this strategy has proven useful when complex admixture shapes the genetic structure $(58,59)$, or when the studied populations are sampled from a small geographic region (19), in which case, defining populations based on geography or culture may lead to a biased view of the genetic structure of the populations. 


\section{Ancestry estimation}

We estimated admixture proportions for each ni-Vanuatu individual using SOURCEFIND (60). We first ran ChromoPainter using the "donors" mode: we considered each ni-Vanuatu individual as a recipient only and defined donor populations by using the genetic clusters defined above (Analysis 2). We ran SOURCEFIND by considering all surrogates ("all surrogates" analysis; Fig. S15 and Table S5) or by limiting as much as possible the number of surrogates ("limited surrogates" analysis). In the latter case, we considered as surrogates West_Eurasia1, West_Eurasia2, West_Eurasia3, EastAsia1, EastAsia2, EastAsia3, Atayal, Paiwan, Southeast_Asia, Cebuano, RenBell, Tikopia, PNG1, PNG2 and PNG_SGDP. Then, we summed the estimated ancestry proportions for these surrogates and grouped them as follows: Taiwanese.Philippines (Atayal, Paiwan, Cebuano), EastAsian_mainland (EastAsia1, EastAsia2, EastAsia3, Southeast_Asia), Polynesian (RenBell, Tikopia), Papuan-related (PNG1, PNG2 and PNG_SGDP) and European (West_Eurasia1, West_Eurasia2, West_Eurasia3). The East Asian-related ancestry was estimated as the sum of Taiwanese.Philippines group and Polynesian group (the EastAsian_mainland was negligible, with a maximum proportion $=0.007)$. To facilitate visualization, we excluded an individual born in Futuna and living in Malekula, who shows a Polynesian ancestry of 0.745 in figures showing ancestry proportions (Fig. 2, Fig. S8-11). The next individual carrying the highest Polynesian ancestry shows 0.383 . For both the "all surrogates" and "limited surrogates" analyses, we ran 200,000 MCMC iterations with a burn-in of 50,000 iterations, sampling every 5,000 iterations and not allowing for self-copy. We estimated the final ancestry of each ni-Vanuatu sample as the mean of the 30 sampled MCMC runs.

\section{Admixture date estimation}

We estimated admixture dates using GLOBETROTTER (61). For this analysis, we ran ChromoPainter using the "donors" mode, by considering the ni-Vanuatu genetic clusters defined in (Analysis 1) as recipients and all the reference populations (Analysis 2) as both recipients and donors. Surrogates were the same as those considered for the "limited surrogates" SOURCEFIND analysis. For each of the 20 recipient ni-Vanuatu clusters, we performed 100 bootstraps, which are implemented as resamplings of chromosomes among the available samples. We set the "Null.ind" option to 1. We assumed a generation time of 28 years, to estimate dates from the number of generations. We also dated admixture in each niVanuatu sample following the same approach. 


\section{Sex-biased admixture}

619

620

We studied sex-biased admixture by comparing ancestry proportions estimated for the autosomes and the $\mathrm{X}$ chromosome. We estimated ancestry proportions using RFMix v.1.5.4 (33), considering as ancestral populations New Guinean highlanders (approximating Papuanrelated ancestry) and Taiwanese Indigenous peoples and the Cebuano from the Philippines (approximating East Asian-related ancestry). We ran the "TrioPhased" algorithm, allowing for phase correction and $3 \mathrm{EM}$ iterations. The window size was set at $0.03 \mathrm{cM}$ (17) and the admixture date was set at 50 generations. The same parameters were used for the $\mathrm{X}$ chromosome and the autosomes. We combined the X chromosome haploid data of males from the same island to obtain diploid individuals. We filtered out SNPs with an RFMix posterior probability $<0.9$, those within centromeres and within $2 \mathrm{Mb}$ from the telomeres. We then estimated East Asian-related and Papuan-related ancestry in the autosomes and the $\mathrm{X}$ chromosome separately. We estimated $\alpha_{f}$ and $\alpha_{m}$, that is, the proportion of female and male ancestors of ni-Vanuatu who carried Papuan-related ancestry, respectively, by assuming that admixture proportions have reached equilibrium values (34). In such a case, $\alpha_{f}=3 \alpha_{X}-$ $2 \alpha_{\text {auto }}$ and $\alpha_{m}=4 \alpha_{\text {auto }}-3 \alpha_{X}$, where $\alpha_{\text {auto }}$ and $\alpha_{X}$ are the average Papuan-related ancestry proportions estimated on the autosomes and the $\mathrm{X}$ chromosome, respectively.

To avoid potential biases due to SNP ascertainment, we also studied sex-biased admixture by comparing the autosomes and the sex chromosomes of 179 ni-Vanuatu sequenced at $30 x$ coverage (17). Because genotypes on the $X$ chromosome were not called in the previous study, we mapped fastq files on the X chromosome of the human reference genome (version hs37d5) and performed genotype calling for X-linked variants, as previously described (17), setting the ploidy parameter to 1 for males and 2 for females. We kept only biallelic SNPs and filtered $v c f$ files following GATK best practices (62): QualByDepth <2; FisherStrand value > 60; StrandOddsRatio > 3; RMSMappingQuality < 40; MappingQualityRankSumTest < -12.5; and ReadPosRankSum $<-8$. We also removed genotypes with a DP $<10$ for females and $<3$ for males, and those with a GQ $<30$. We removed PAR1, PAR2 and XTR regions according to the annotations provided by the UCSC browser, and amplicon regions as reported in (63). We filtered out SNPs that were not in Hardy-Weinberg equilibrium in females $(P$-value $<$ 0.0001 ), those with a missingness $>0.05$ and those with a MAF $<0.01$. We estimated mtDNA haplogroups with MToolBox (64) from the bam and fastq files, and Y chromosome haplogroups were estimated with Yleaf (65). 


\section{Inferring migrations within Vanuatu}

652

653

654

We used the genetic structure inferred by fineSTRUCTURE to study migration patterns among Vanuatu islands. First, we assigned each genetic cluster to one or more islands, each time more than $25 \%$ of the sampled individuals inhabiting the island were assigned to that cluster. We then identified "outlier" individuals who inhabit an island but are assigned to a genetic cluster that is prevalent in another island. These individuals, either themselves or their close ancestors, likely migrated from the island/s where their genetic cluster is predominant to their current place of residence. We removed from these analyses Vanuatu_8 and Vanuatu_9 clusters $\left(K_{\mathrm{FS}}=20\right)$ because those clusters are driven by recent European gene flow, as well as Vanuatu_15 because no island could be assigned to this cluster when using the $25 \%$ rule. To study sex-specific migrations, we calculated the proportion of female (male) migrants by dividing the number of "outlier" females (males) by the number of females (males) in each cluster. We did not include clusters where the number of males or females was $<2$.

\section{Tests for exogamy}

We tested if spouses show lower genetic relatedness than non-spouses by testing if the average kinship coefficient between spouses is higher or lower than expected by chance, using either permutations or a logistic regression model. When using permutations, we accounted for isolation by distance by sampling random pairs of males and females among individuals born in the same island (Fig. S24A) or living in the same village (Fig. S24B). Because marriages between first-degree related individuals are very unlikely (they are indeed not observed in the data), we excluded from this analysis individuals who are first-degree relatives, but also tested how the inclusion of these individuals affect the results (Fig. S24C). We performed 10,000 permutations for each island. In each permutation, we sampled equally many random pairs as there were observed pairs in the island. The null distribution was built by estimating the average kinship coefficient among randomly sampled pairs, using KING v.2.1. We calculated $P$-values by comparing the average kinship coefficient among the observed couples to the null distribution.

Based on the sample scheme described above, we designed a logistic regression model that could be generalized to multiple explanatory variables, such as ancestry. Let the variable $i$ index all possible pairs of males and females in the dataset, except pairs of individuals who are first-degree relatives, and define the dependent binary variable $Y_{i}$ with $Y_{i}=1$ if $i$ indexes an observed pair of spouses and $Y_{i}=0$ otherwise. Define the probability that a pair is an 
687

observed couple $p_{i}(x)=P\left(Y_{i}=1\right)$, and introduce $\phi_{i}$ as the kinship coefficient between individuals in the $i$ th pair. We estimated the effect of kinship on mate choice by the parameter $\beta^{\phi}$ in the logistic regression model

$$
\log \left[\frac{p_{i}(x)}{1-p_{i}(x)}\right]=\mu+\phi_{i} \beta^{\phi}+I\left(\text { village }_{i}\right) \beta^{v}+I\left(\text { island }_{i}\right) \beta^{I}
$$

where $I\left(\right.$ village $\left._{i}\right)=1$ if individuals of pair $i$ are from the same village and $I$ (village $\left.e_{i}\right)=0$ otherwise, and $I\left(\right.$ island $\left._{i}\right)=1$ if individuals of pair $i$ are from the same island and $I\left(\right.$ island $\left._{i}\right)=0$ otherwise. As a sensitivity analysis, we also considered a model in which pairs of first-degree related individuals were included (Fig. S25).

\section{Tests for ancestry-based assortative mating}

We extended the logistic regression model shown in Eq. 1 to test for ancestry-based assortative mating, by testing if the genetic ancestry of spouses is more similar than that of non-spouses, accounting for population structure and relatedness avoidance. Let $q_{i 1}^{\text {EA }}$ and $q_{i 2}^{\text {EA }}$ be the proportion of East Asian-related ancestry for the individuals 1 and 2 of pair $i$ and introduce the variable $q_{i}^{E A}=\left|q_{i 1}^{\mathrm{EA}}-q_{i 2}^{\mathrm{EA}}\right|$.We estimated the effect of having similar proportions of East Asian-related ancestry between two individuals on the probability that a pair is an observed couple $p_{i}(x)$ by the parameter $\beta^{q}$ in the logistic regression model,

$$
\log \left[\frac{p_{i}(x)}{1-p_{i}(x)}\right]=\mu+q_{i}^{E A} \beta^{q}+\phi_{i} \beta^{\phi}+I\left(\text { village }_{i}\right) \beta^{v}+I\left(\text { island }_{i}\right) \beta^{I},
$$

where we use the same notations as in Eq. 1. A negative (positive) effect size $\beta^{q}$ is interpreted as evidence for assortative (disassortative) mating according to ancestry. Effect sizes for other ancestries were calculated similarly. The same model was also tested in ni-Vanuatu originating or not from islands where Polynesian languages are spoken. Note that in standard regression analyses, such as those used in GWAS, population stratification is usually corrected by principal components of the genetic relatedness matrix; here we have taken a more general approach by decomposing the genetic structure in two variables: the kinship and the ancestry, and have studied how both variables affect mate choice independently.

\section{Tests for SNP-based assortative mating}


712 We extended the logistic regression model shown in Eq. 1 to test for SNP-based assortative mating, by testing if the genotypes at a given SNP are more similar between spouses than non-spouses, accounting for population structure, ancestry-based associated mating and relatedness avoidance. Define, for each SNP $s$ and each pair $i$ of individuals, the allele sharing distance (ASD) $d_{i, s}$ as $d_{i, s}=0$ if both alleles are identical between individuals, $d_{i, s}=1$ if only one allele is identical and $d_{i, s}=2$ if none of the alleles are identical. We

$$
\log \left[\frac{p_{i}(x)}{1-p_{i}(x)}\right]=\mu+d_{i, s} \beta_{s}^{A S D}+q_{i}^{E A} \beta^{q}+\phi_{i} \beta^{\phi}+I\left(\text { village }_{i}\right) \beta^{v}+I\left(\text { island }_{i}\right) \beta^{I},
$$

720

721

where we use the same notations as in Eq. 2. A negative (positive) effect size $\beta_{s}^{A S D}$ indicates higher (lower) similarity at SNP $s$ between the members of the observed couples than between non-couples, in line with SNP-based assortative (disassortative) mating.

\section{Tests for trait-based assortative mating}

We tested for trait-based assortative mating by testing if genotypes of spouses are more similar or dissimilar at trait-associated SNPs, relative to non-associated SNPs. We obtained GWAS summary statistics for 8 candidate traits from the UK Biobank database (http://www.nealelab.is/uk-biobank). Candidate traits include traits relating to morphology and physical appearance. Let $y_{S}=\beta_{S}^{A S D}$, where $\beta_{S}^{A S D}$ is the effect size of SNP $s$ on mate choice estimated by Eq. 1. We estimated if trait-associated SNPs are more similar or dissimilar between spouses by the parameter $\beta^{\text {trait }}$ in the model

$$
y_{s}=\mu+I\left(\text { associated }_{s}\right) \beta^{\text {trait }}+M A F_{s} \beta^{M A F}+G E R P_{s} \beta^{G E R P}+\operatorname{rec}_{s} \beta^{\text {rec }} \text {, }
$$

where $I$ (associated $\left._{s}\right)=1$ if SNP $s$ is significantly associated with the candidate trait in GWAS (with GWAS $P$-value $<5 \times 10^{-8}$ ) and $I\left(\right.$ associated $\left._{S}\right)=0$ otherwise, $M A F_{S}$ is the minor allele frequency of SNP $s$ in ni-Vanuatu, GERP $P_{s}$ is the Genomic Evolutionary Rate Profiling (GERP) score of SNP $s$ and $r e c_{s}$ is the interpolated recombination rate between SNP $s$ and SNP $s-1$, estimated in $\mathrm{cM} / \mathrm{Mb}$ from the 1000 Genomes Phase 3 combined recombination map. We considered that there is trait-based assortative (disassortative) mating 
bioRxiv preprint doi: https://doi.org/10.1101/2022.01.10.475623; this version posted January 10,2022 . The copyright holder for this preprint (which was not certified by peer review) is the author/funder, who has granted bioRxiv a license to display the preprint in perpetuity. It is made available under aCC-BY-NC-ND 4.0 International license.

741 if $\beta^{\text {trait }}$ is significantly negative (positive). We adjusted $P$-values with the Bonferroni

742 correction for multiple testing, to account for the number of traits tested.

743 


\section{Acknowledgments}

745 We thank all volunteers in Vanuatu who kindly provided samples and participated in this

746 research. We thank the Ministry of Health of the Republic of Vanuatu (especially Myriam

747 Abel, Rose Bahor, Yvannah Taga, Maturine Tary and the former Minister Jerôme Ludvaune),

748 the Vanuatu Family Health Association (especially Blandine Boulekone and Marie Nickllum Wala), the WHO Office in Port Vila (especially Corinne Capuano) and the Institut Pasteur of New Caledonia at Nouméa (especially Paul M. V. Martin and Eliane Chungue), for their continuous support and interest in this work. We are particularly thankful to Helene Walter, Woreka Mera, Françoise Charavay and Sonia Treptow for their assistance in collecting samples. We thank S. Créno and the HPC Core Facility of Institut Pasteur (Paris) for the management of computational resources. L.R.A. was funded by a Pasteur-Roux-Cantarini fellowship from the Institut Pasteur. The laboratory of Human Evolutionary Genetics is supported by the Institut Pasteur, the Collège de France, the CNRS, the Fondation AllianzInstitut de France, the French Government's Investissement d'Avenir programme, Laboratoires d'Excellence 'Integrative Biology of Emerging Infectious Diseases' (ANR-10LABX-62-IBEID) and 'Milieu Intérieur' (ANR-10-LABX-69-01), the Fondation de France $\left(\mathrm{n}^{\circ} 00106080\right)$, and the Fondation pour la Recherche Médicale (Equipe FRM DEQ20180339214) and the French National Research Agency (ANR-19-CE35-0005).

Authors contributions: L.R.A, E.P. and L.Q.-M. designed research; L.R.A analyzed data, with input from J.B., J.C., J.M.R., M.R. and E.P. C.H. and L.L. performed DNA extractions and genotyping. A.M.S., H.C., A.F., F.V., O.C., and A.G. provided resources, expertise and feedback. L.Q.-M and E.P. supervised the study. L.Q.M secured funding. L.R.A., L.Q.M. and E.P. wrote the paper, with input from all authors.

Competing Interest Statement: The authors declare no competing interests. 


\section{References}

773 1. Bellwood P (2004) First Farmers: The Origins of Agricultural Societies (Wiley-Blackwell).

2. Gray RD, Drummond AJ, \& Greenhill SJ (2009) Language phylogenies reveal expansion pulses and pauses in Pacific settlement. Science 323(5913):479-483.

3. Hung H-C \& Carson MT (2015) Foragers, fishers and farmers: origins of the Taiwanese Neolithic. Antiquity 88(342):1115-1131.

4. Kirch PV (2017) On the road of the winds: An archeological history of the Pacific islands before European contact (University of California Press).

5. Sheppard PJ (2011) Lapita Colonization across the Near/Remote Oceania Boundary. Curr Anthropol 52(6):799-840.

6. Skoglund P, et al. (2016) Genomic insights into the peopling of the Southwest Pacific. Nature 538(7626):510-513.

7. Valentin F, Detroit F, Spriggs MJ, \& Bedford S (2016) Early Lapita skeletons from Vanuatu show Polynesian craniofacial shape: Implications for Remote Oceanic settlement and Lapita origins. Proc Natl Acad Sci U S A 113(2):292-297.

8. Lipson M, et al. (2018) Population Turnover in Remote Oceania Shortly after Initial Settlement. Curr Biol 28(7):1157-1165 e1157.

9. Posth C, et al. (2018) Language continuity despite population replacement in Remote Oceania. Nat Ecol Evol 2(4):731-740.

10. Kirch PV (1984) The Polynesian outliers: Continuity, change, and replacement. J Pac Hist 19:224-238.

11. Feinberg RS (2012) Polynesian Outliers: The State of the Art (University of Pittsburgh).

12. Lipson M, et al. (2020) Three Phases of Ancient Migration Shaped the Ancestry of Human Populations in Vanuatu. Curr Biol 30(24):4846-4856 e4846.

13. François A, Lacrampe S, Franjieh M, \& Schnell S (2015) The Languages of Vanuatu: Unity and Diversity (Canberra: Asia-Pacific Linguistics).

14. Heyer E, Chaix R, Pavard S, \& Austerlitz F (2012) Sex-specific demographic behaviours that shape human genomic variation. Mol Ecol 21(3):597-612.

15. Zou JY, et al. (2015) Genetic and socioeconomic study of mate choice in Latinos reveals novel assortment patterns. Proc Natl Acad Sci U S A 112(44):13621-13626.

16. Ceballos FC, Joshi PK, Clark DW, Ramsay M, \& Wilson JF (2018) Runs of homozygosity: windows into population history and trait architecture. Nat Rev Genet 19(4):220-234.

17. Choin J, et al. (2021) Genomic insights into population history and biological adaptation in Oceania. Nature 592(7855):583-589.

18. Lawson DJ, Hellenthal G, Myers S, \& Falush D (2012) Inference of population structure using dense haplotype data. PLoS Genet 8(1):e1002453. 
19. Leslie S, et al. (2015) The fine-scale genetic structure of the British population. Nature 519(7543):309-314.

20. van Dorp L, et al. (2015) Evidence for a Common Origin of Blacksmiths and Cultivators in the Ethiopian Ari within the Last 4500 Years: Lessons for Clustering-Based Inference. PLoS Genet 11(8):e1005397.

21. Tryon DT (1976) New Hebrides Languages: An Internal Classification (Australian National University, Canberra) p 545.

22. François A (2011) Where *R they all? The Geography and History of *R-loss in Southern Oceanic Languages. Oceanic Linguistics 50(1):140-197.

23. Lynch JD, Ross MT, \& Crowley T (2011) The Oceanic Languages (Routledge).

24. Crowley T (2000) The Language Situation in Vanuatu. Current Issues in Language Planning 1(1).

25. Hau'ofa E (1994) Our Sea of Islands. The Contemporary Pacific 6(1):148-161.

26. Hermann A \& Walworth M (2020) Approche interdisciplinaire des échanges interculturels et de l'intégration des communautés polynésiennes dans le centre du Vanuatu. Journal de la société des océanistes 151:239-262.

27. Lynch JD \& Fakamuria K (1994) Borrowed Moieties, Borrowed Names: Sociolinguistic Contact between Tanna and Futuna-Aniwa, Vanuatu. Pacific Studies 17(1):79-91.

28. Bedford S \& Spriggs M (2008) Northern Vanuatu as a Pacific Crossroads: The Archaeology of Discovery, Interaction, and the Emergence of the "Ethnographic Present". Asian Perspectives 47(1):95-120.

29. Monzier M, Robin C, \& Eissen J-P (1994) Kuwae ( $\approx 1425$ A.D.): the forgotten caldera. $J$ Volcanol Geoth Res 59(3):207-218.

30. Gao C, et al. (2006) The 1452 or 1453 A.D. Kuwae eruption signal derived from multiple ice core records : Greatest volcanic sulfate event of the past 700 years. J Geophysical Res 111(D12107):111.

31. Flexner J, Spriggs M, Bedford S, \& Abong M (2016) Beginning Historical Archaeology in Vanuatu: Recent Projects on the Archaeology of Spanish, French, and Anglophone Colonialism. Archaeologies of Early Modern Spanish Colonialism, (Springer International Publishing), pp 205227.

32. Jolly M (2009) The Sediment of Voyages: Re-membering Quirós, Bougainville and Cook in Vanuatu. Oceanic Encounters: Exchange, Desire, Violence, (ANU Press), pp 57-112.

33. Maples BK, Gravel S, Kenny EE, \& Bustamante CD (2013) RFMix: a discriminative modeling approach for rapid and robust local-ancestry inference. Am J Hum Genet 93(2):278-288.

34. Goldberg A \& Rosenberg NA (2015) Beyond 2/3 and 1/3: The Complex Signatures of Sex-Biased Admixture on the X Chromosome. Genetics 201(1):263-279.

35. Vienne B (1984) Gens de Motlav. Idéologie et pratique sociale en Mélanésie. Publication de la Société des Océanistes 42:232-240. 
36. Bolton L (1999) Women, Place and Practice in Vanuatu: a View from Ambae. Oceania 70:43-55.

37. Petrou K \& Connell J (2017) Rural-urban migrants, translocal communities and the myth of return migration in Vanuatu: the case of Paama. Journal de la société des Océanistes 144-145(1-2):5162.

38. Godelier M (2004) Métamorphoses de la parenté (Fayard, Paris).

39. Parra FC, et al. (2003) Color and genomic ancestry in Brazilians. Proc Natl Acad Sci U S A 100(1):177-182.

40. Alvarez L \& Jaffe K (2004) Narcissism guides mate selection: Humans mate assortatively, as revealed by facial resemblance, following an algorithm of "self seeking like". Evol Psychol 2:177194.

41. Pugach I, et al. (2018) The gateway from Near into Remote Oceania: new insights from genomewide data. Mol Biol Evol 35(4):871-886.

42. Flexner JL, Bedford S, \& Valentin F (2019) Who was Polynesian? Who was Melanesian? Hybridity and ethnogenesis in the South Vanuatu Outliers. J Social Archaeol 19:403-426.

43. Bonnemaison J (1996) Arts of Vanuatu (University of Hawaii Press).

44. Kirin M, et al. (2010) Genomic runs of homozygosity record population history and consanguinity. PLoS One 5(11):e13996.

45. McQuillan R, et al. (2008) Runs of homozygosity in European populations. Am J Hum Genet 83(3):359-372.

46. Bianco E, et al. (2020) Recent Common Origin, Reduced Population Size, and Marked Admixture Have Shaped European Roma Genomes. Mol Biol Evol 37(11):3175-3187.

47. Ruiz-Linares A, et al. (2014) Admixture in Latin America: geographic structure, phenotypic diversity and self-perception of ancestry based on 7,342 individuals. PLoS Genet 10(9):e1004572.

48. Chang CC, et al. (2015) Second-generation PLINK: rising to the challenge of larger and richer datasets. Gigascience 4:7.

49. Manichaikul A, et al. (2010) Robust relationship inference in genome-wide association studies. Bioinformatics 26(22):2867-2873.

50. Mallick S, et al. (2016) The Simons Genome Diversity Project: 300 genomes from 142 diverse populations. Nature 538(7624):201-206.

51. Delaneau O, Marchini J, \& Zagury JF (2011) A linear complexity phasing method for thousands of genomes. Nat Methods 9(2):179-181.

52. Delaneau O, Zagury JF, \& Marchini J (2013) Improved whole-chromosome phasing for disease and population genetic studies. Nat Methods 10(1):5-6.

53. Auton A, et al. (2015) A global reference for human genetic variation. Nature 526(7571):68-74.

54. Patterson N, Price AL, \& Reich D (2006) Population structure and eigenanalysis. PLoS Genet 2(12):e190. 
55. Alexander DH, Novembre J, \& Lange K (2009) Fast model-based estimation of ancestry in unrelated individuals. Genome Res 19(9):1655-1664.

56. Behr AA, Liu KZ, Liu-Fang G, Nakka P, \& Ramachandran S (2016) pong: fast analysis and visualization of latent clusters in population genetic data. Bioinformatics 32(18):2817-2823.

57. Pembleton LW, Cogan NO, \& Forster JW (2013) StAMPP: an R package for calculation of genetic differentiation and structure of mixed-ploidy level populations. Mol Ecol Resour 13(5):946-952.

58. Arauna LR, et al. (2017) Recent Historical Migrations Have Shaped the Gene Pool of Arabs and Berbers in North Africa. Mol Biol Evol 34(2):318-329.

59. Mas-Sandoval A, et al. (2019) Reconstructed Lost Native American Populations from Eastern Brazil Are Shaped by Differential Je/Tupi Ancestry. Genome Biol Evol 11(9):2593-2604.

60. Chacon-Duque JC, et al. (2018) Latin Americans show wide-spread Converso ancestry and imprint of local Native ancestry on physical appearance. Nat Commun 9(1):5388.

61. Hellenthal G, et al. (2014) A genetic atlas of human admixture history. Science 343(6172):747751.

62. Van der Auwera GA, et al. (2013) From FastQ data to high confidence variant calls: the Genome Analysis Toolkit best practices pipeline. Curr Protoc Bioinformatics 43:11 10 11-11 1033.

63. Lucotte EA, et al. (2018) Dynamic Copy Number Evolution of X-and Y-Linked Ampliconic Genes in Human Populations. Genetics 209(3):907-920.

64. Calabrese C, et al. (2014) MToolBox: a highly automated pipeline for heteroplasmy annotation and prioritization analysis of human mitochondrial variants in high-throughput sequencing. Bioinformatics 30(21):3115-3117.

65. Ralf A, Montiel Gonzalez D, Zhong K, \& Kayser M (2018) Yleaf: Software for Human YChromosomal Haplogroup Inference from Next-Generation Sequencing Data. Mol Biol Evol 35(5):1291-1294. 


\section{$908 \quad$ Figure legends}

909 Fig. 1. Sampling locations and genetic structure in Vanuatu. (A) Map showing the location of 910 the 1,439 sampled ni-Vanuatu individuals. The inset at the bottom left shows the location of 911 Vanuatu in the Asia-Pacific region. $(B, C)$ Principal Component Analysis (PCA) of genotypes 912 of ni-Vanuatu at 301,774 SNPs, in the context of $(B)$ the broad Asia-Pacific region and $(C)$ the 913 Vanuatu archipelago only. (A-C) Each point indicates an individual, colored according to the 914 latitude of their island of residence.

Fig. 2. Fine-scale genetic structure and admixture in Vanuatu. (A) Map showing the clustering of 979 ni-Vanuatu into 20 genetic clusters, according to fineSTRUCTURE $\left(K_{\mathrm{FS}}=20\right)$. Each point indicates an individual, located according to their village of residence. Colors indicate genetic clusters, so that the closer the colors, the closer the clusters. Noise was added to sampling locations to facilitate visualization. (B) Admixture date estimates for each genetic cluster, based on 100 bootstrap replicates. Colors are the same as those used in $(A)$. The $\mathrm{x}$-axis shows the admixture date in years before present, assuming a generation time of 28 years. The $y$-axis indicates the latitude of the islands assigned to each genetic cluster. The heights of the density curves are proportional to the sample size of each cluster. $(C)$ Proportion of East Asian-related ancestry estimated by SOURCEFIND. (D) Ratio of the Polynesian and East Asian-related ancestry proportions, estimated by SOURCEFIND. The inset at the bottom left shows the location of Polynesian-speaking individuals, indicated by black points. $(C-D)$ Each point indicates an individual, colored according to their ancestry proportions.

Fig 3. Sex-biased admixture and migration patterns in Vanuatu. (A) Papuan-related ancestry proportions in ni-Vanuatu, estimated for the 22 autosomes and the $\mathrm{X}$ chromosome separately by RFMix. (B) Frequencies of Y chromosome and mtDNA haplogroups, colored according to their assumed origins (i.e., shades of blue or red indicate Papuan- or East Asian-related origins, respectively). Haplogroups were inferred from high-coverage genome sequencing data obtained for a subset of 179 ni-Vanuatu (17). (C) Recent migrations among Vanuatu islands, inferred based on fineSTRUCTURE clusters. The arrows connect the location of the genetic cluster to which individuals were assigned to their actual place of residence. The colors indicate the predominant genetic cluster in the island of origin. The width is proportional to the number of inferred migrant individuals, relative to the number of females or males in the genetic cluster. 
942 Fig 4. Ancestry- and trait-based assortative mating in Vanuatu. (A) Effects of kinship and

943 ancestry differences on partner choice. Effect sizes were estimated with a logistic regression

944 model, while accounting for population structure (island of birth and village of residence).

945 The effect size and $P$-value were estimated using different ancestries as predictors,

946 independently. (B) Increased (purple) or decreased (orange) genetic similarity among spouses

947 (as measured by $\beta_{S}^{A S D}$ ) at trait-associated SNPs, relative to non-associated SNPs. Results on

948 the left are based on a logistic model that includes only the genotype dissimilarity among

949 spouses at each SNP, whereas results on the right are based on the logistic model that also

950 controls for possible confounders (i.e. geography, kinship and ancestry differences). 


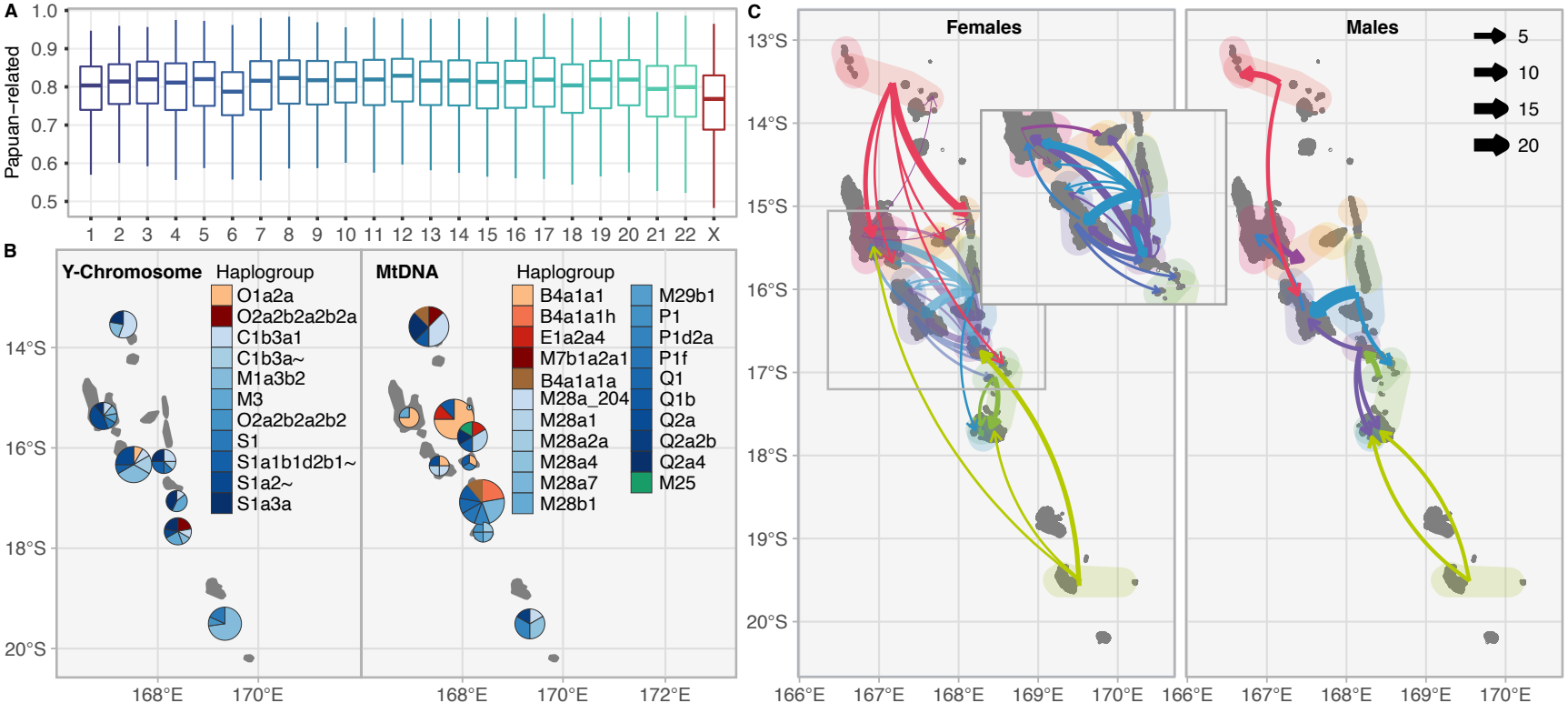


A

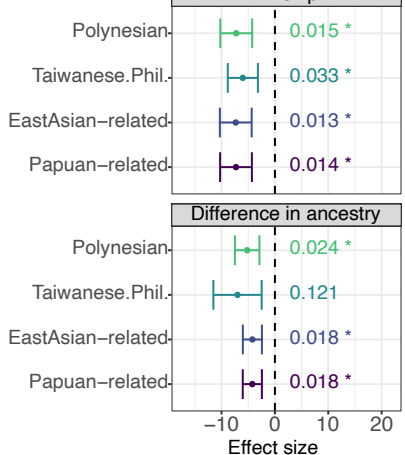

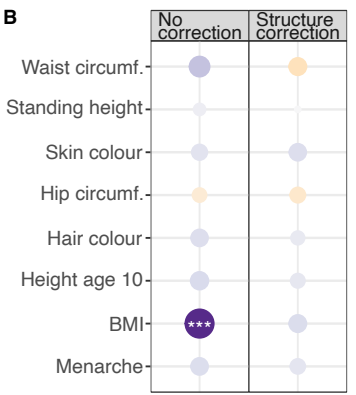

Effect size for $\beta_{A S D}$

$\begin{array}{lllll}-0.008 & -0.004 & 0 & 0.004 & 0.008\end{array}$ 